\title{
Variation in Screening Abnormality Rates and Follow-Up of Breast, Cervical and Colorectal Cancer Screening within the PROSPR Consortium
}

\author{
Anna N. A. Tosteson, ScD 1,2, Elisabeth F. Beaber, $P h D, M P H^{3}$, Jasmin Tiro, $P h D^{4}$, Jane Kim, $P h D^{5}$, \\ Anne Marie McCarthy, $P h D^{6}$, Virginia P. Quinn, $P h D, M P H^{7}$, V. Paul Doria-Rose, DVM, PhD ${ }^{8}$, \\ Cosette M. Wheeler, PhD ${ }^{9}$, William E. Barlow, $P h D^{10}$, Mackenzie Bronson, BA ${ }^{7}$, Michael Garcia, $\mathrm{MS}^{3}$, \\ Douglas A. Corley, MD, PhD, MPH ${ }^{17}$, Jennifer S. Haas, MD, MSc ${ }^{12}$, Ethan A. Halm, MD, MPH ${ }^{13}$, \\ Aruna Kamineni, $\mathrm{PhD}, \mathrm{MPH}^{14}$, Carolyn M. Rutter, $\mathrm{PhD}{ }^{15}$, Tor D. Tosteson, $\mathrm{ScD}{ }^{1,2}$, \\ Amy Trentham-Dietz, $P h D^{16}$, and Donald L. Weaver, $M D^{17}$ on behalf of the PROSPR consortium \\ ${ }^{1}$ Geisel School of Medicine at Dartmouth, Lebanon, NH, USA; ${ }^{2}$ Norris Cotton Cancer Center, Lebanon, NH, USA; ${ }^{3}$ Fred Hutchinson Cancer Research \\ Center, Seattle, WA, USA; ${ }^{4}$ University of Texas Southwestern, Dallas, TX, USA; ${ }^{5}$ Harvard T.H. Chan School of Public Health, Boston, MA, USA; \\ ${ }^{6}$ Massachusetts General Hospital, Boston, MA, USA; ${ }^{7}$ Kaiser Permanente Southern California, Pasadena, CA, USA; ${ }^{8}$ National Cancer Institute,

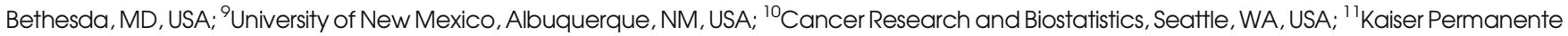 \\ Northern California, Oakland, CA, USA; ${ }^{12}$ Brigham and Women's Hospital, Boston, MA, USA; ${ }^{13}$ University of Texas Southwestern Medical Center, \\ Dallas, TX, USA; ${ }^{14}$ Group Health, Seattle, WA, USA; ${ }^{15}$ RAND Corporation, Santa Monica, CA, USA; ${ }^{16}$ University of Wisconsin, Madison, WI, USA; \\ ${ }^{17}$ University of Vermont, Burlington, VT, USA.
}

BACKGROUND: Primary care providers and health systems have prominent roles in guiding effective cancer screening.

OBJECTIVE: To characterize variation in screening abnormality rates and timely initial follow-up for common cancer screening tests.

DESIGN: Population-based cohort undergoing screening in 2011, 2012, or 2013 at seven research centers comprising the National Cancer Institute-sponsored Population-based Research Optimizing Screening through Personalized Regimens (PROSPR) consortium.

PARTICIPANTS: Adults undergoing mammography with or without digital breast tomosynthesis ( $n=97,683$ ages 40-75 years), fecal occult blood or fecal immunochemical tests ( $n=759,553$ ages 50-75 years), or Papanicolaou with or without human papillomavirus tests ( $n=167,330$ ages 21-65 years).

INTERVENTION: Breast, colorectal, or cervical cancer screening.

MAIN MEASURES: Abnormality rates per 1000 screens; percentage with timely initial follow-up (within 90 days, except 9-month window for BI-RADS 3). Primary care clinic-level variation in percentage with screening abnormality and percentage with timely initial follow-up.

KEY RESULTS: There were 10,248/97,683 (104.9 per 1000) abnormal breast cancer screens, 35,847/759,553 (47.2 per 1000) FOBT/FIT-positive colorectal cancer screens, and 13,266/167,330 (79.3 per 1000) abnormal cervical cancer screens. The percentage with timely follow-up was 93.2 to $96.7 \%$ for breast centers, 46.8 to $68.7 \%$ for colorectal centers, and $46.6 \%$ for the cervical

Electronic supplementary material The online version of this article (doi:10.1007/s11606-015-3552-7) contains supplementary material, which is available to authorized users.

Published online December 9, 2015 cancer screening center (low-grade squamous intraepithelial lesions or higher). The primary care clinic variation (25th to 75 th percentile) was smaller for the percentage with an abnormal screen (breast, 8.5-10.3\%; colorectal, 3.0-4.8\%; cervical, 6.3-9.9\%) than for the percentage with follow-up within 90 days (breast, 90.2-95.8 \%; colorectal, 43.4-52.0\%; cervical, 29.6-61.4\%).

CONCLUSIONS: Variation in both the rate of screening abnormalities and their initial follow-up was evident across organ sites and primary care clinics. This highlights an opportunity for improving the delivery of cancer screening through focused study of patient, provider, clinic, and health system characteristics associated with timely follow-up of screening abnormalities.

KEY WORDS: breast cancer screening; colorectal cancer screening; cervical cancer screening; practice variation.

J Gen Intern Med 31(4):372-9

DOI: $10.1007 / \mathrm{s} 11606-015-3552-7$

(c) Society of General Internal Medicine 2015

S creening for breast, colorectal, and cervical cancer is an integral component of preventive health care in the United States. The importance of cancer screening is underscored by its mandated insurer coverage in the Affordable Care Act ${ }^{1}$ and the creation of screening guidelines by the United States Preventive Services Task Force (USPSTF), which provides evidence-based guidelines for average-risk populations. ${ }^{2-4}$

In 2011, the U.S. National Cancer Institute (NCI) established the Population-based Research Optimizing Screening through Personalized Regimens (PROSPR) consortium, funding seven research centers, with an overall goal of promoting 
coordinated, multidisciplinary research to evaluate and improve the cancer screening process across the care continuum from cancer screening to diagnosis and treatment. An essential premise in establishing PROSPR, initially comprised of three centers focused on breast cancer, three on colorectal cancer, and one on cervical cancer screening, is that variation at multiple levels - health system, clinic, provider, and patient - may be leveraged through comparative effectiveness research to elucidate best cancer screening practices. To advance research in this area, the consortium has developed conceptual models for studying screening processes of care ${ }^{5-}$ ${ }^{7}$ that extend the work of Zapka and Taplin. ${ }^{8}$ These models detail the screening process from risk assessment through diagnosis and treatment as steps with interfaces requiring a transfer of information and/or responsibility across providers, and elucidate transitions where threats to timely follow-up may occur at multiple levels. ${ }^{5}$

The purpose of this paper is to describe observed variations in rates of screening abnormalities and their timely follow-up, with attention to similarities and differences across breast, cervical, and colorectal organ sites and to variations across primary care clinics. With primary care providers and health care systems increasingly taking responsibility for population health through the emergence of patient-centered medical homes and accountable care organizations, ${ }^{9}$ consideration of variation in cancer screening practices from a primary health care delivery perspective may shed light on areas where the quality of cancer screening can be improved. ${ }^{10,11}$ Focusing on the patient and their interaction with the entirety of cancer screening, beyond organ-centric cancer screening, has the potential to improve overall quality of care. ${ }^{5,12,13}$

\section{METHODS}

This study was conducted as part of the NCI-funded Population-Based Research Optimizing Screening through Personalized Regimens (PROSPR) consortium. The overall aim of PROSPR is to conduct multi-site, coordinated, transdisciplinary research to evaluate and improve cancer screening processes. The seven PROSPR research centers reflect the diversity of U.S. health care delivery organizations. The three breast centers include an integrated health care delivery system affiliated with the University of Pennsylvania (UPenn), a statewide mammography and pathology registry housed at the University of Vermont (VT), and primary care practice networks in two states within the Dartmouth-Hitchcock health system in New Hampshire and Brigham and Women's Hospital in Massachusetts (Dartmouth-BWH). The three integrated health care systems comprising the colorectal centers include two managed care plans, Kaiser Permanente Northern and Southern California (KP) and Group Health (GH), and a regional safety-net clinical provider network at the ParklandUniversity of Texas Southwestern Medical Center (Parkland-
UTSW). The cervical center is a statewide registry housed at the University of New Mexico (NM). Each center submits common data elements obtained from several sources (e.g., electronic health records, administrative data) to a central data repository, which undergoes rigorous data quality assurance processes involving harmonization to account for differences in data sources.

\section{Study Populations}

Individuals undergoing screening with the tests of interest in 2011, 2012, or 2013 who met commonly used age criteria for screening were studied. ${ }^{2-4}$ For breast cancer screening, we report on women aged 40-75 years screened in 2012 or 2013 , with no prior history of breast cancer, who received screening digital mammography with or without digital breast tomosynthesis and had no breast imaging in the prior 90 days. For cervical cancer screening, women aged 21-65 years who received Papanicolaou (Pap)-stained cervical cytology with or without human papillomavirus (HPV) tests in 2012 and had no Pap test in the prior 300 days were studied. For colorectal cancer screening, we report on men and women aged 50-75 years who received high-sensitivity guaiac-based fecal occult blood tests (FOBT) or fecal immunochemical tests (FIT) in 2011. Activities were approved by the institutional review boards at each research center and by the PROSPR Statistical Coordinating Center.

\section{Screening Abnormalities and Timely Initial Follow-Up of Abnormalities}

The screening abnormalities studied and expected time frame for timely follow-up are summarized in Table 1. Due to current PROSPR data availability, we restricted reporting for cervical cancer screening to the subset of abnormalities where followup is anticipated within 3 months. This includes the combination of HPV+ and atypical squamous cells of undetermined significance (ASC-US), and low-grade squamous intraepithelial lesion (LSIL) or higher-grade lesions (ASC-H: atypical squamous cells, cannot exclude high-grade squamous intraepithelial lesion, and HSIL: high-grade squamous intraepithelial lesion).

\section{Statistical Analysis}

We report screening abnormality rates per 1000 by organ site and center. For the centers that report screening data by primary care clinic (UPenn, Dartmouth-BWH, Parkland-UTSW, GH, NM), we describe the distribution of screening abnormalities across clinics within centers using medians and interquartile ranges (IQR), and portray these graphically using box plots. For cervical cancer screening, we included all submitted Pap tests regardless of provider type. Due to the instability of rates in light of the small sample sizes, clinic-level analyses are restricted to clinics with 25 or more screening exams for abnormality rates and to 11 or more abnormal screening exams for follow-up rates. 
Table 1 Definitions for Abnormal Screening and Timely Follow-Up Testing

\begin{tabular}{|c|c|c|c|}
\hline Cancer type and screening test & Screening abnormality & $\begin{array}{l}\text { Type of follow-up testing or } \\
\text { diagnostic work-up }\end{array}$ & $\begin{array}{l}\text { Definition of timely } \\
\text { follow-up }\end{array}$ \\
\hline Breast* - Mammography, & BI-RADS 0 & Additional imaging & Within 3 monthst \\
\hline \multirow[t]{2}{*}{ Digital breast tomosynthesis } & BI-RADS 3 & Additional imaging & Within 9 months $\dagger$ \\
\hline & BI-RADS 4, 5 & Additional imaging or biopsy & Within 3 months $\dagger$ \\
\hline Colorectal - FOBT/FIT & FOBT+ or FIT+ & Colonoscopy & Within 3 months \\
\hline \multirow{10}{*}{ Cervical $\ddagger$ - Pap, HPV } & Pap-/HPV+ & Repeat co-testing, or & Within 12 months \\
\hline & & HPV 16 or $16 / 18$ testing & Within 1 month \\
\hline & & -If negative, repeat co-test & Within 12 months \\
\hline & & -If positive, colposcopy \pm biopsy & Within 3 months \\
\hline & ASC-US/HPV- & Repeat Pap, or & Within $12-36$ months \\
\hline & & Co-test & Within 63 months \\
\hline & ASC-US/No HPV & Pap, or & Within 6 months \\
\hline & & Colposcopy \pm biopsy & Within 3 months \\
\hline & ASC-US/HPV+ & Colposcopy \pm biopsy & Within 3 months $\dagger$ \\
\hline & $\begin{array}{l}\text { LSIL, ASC-H, or HSIL; } \\
\text { any HPV result }\end{array}$ & Colposcopy \pm biopsy & Within 3 months $\dagger$ \\
\hline
\end{tabular}

* Breast Imaging-Reporting and Data System (BI-RADS). Additional imaging includes mammogram, digital breast tomosynthesis, ultrasound, or MRI FFollow-up reported on in this paper

$\ddagger$ Human papillomavirus (HPV), atypical squamous cells of undetermined significance (ASC-US), low-grade squamous intraepithelial lesion (LSIL), atypical squamous cells, cannot exclude high-grade squamous intraepithelial lesions (ASC-H), high-grade squamous intraepithelial lesions (HSIL)

We portray time to initial follow-up of an abnormal screening test using Kaplan-Meier plots by center. To characterize variation in timely follow-up, we report both the overall percentage of cases with timely follow-up across centers within organ and by clinic, and the median days and IQR from abnormal result to follow-up.

We used generalized linear mixed models to determine whether observed variation in follow-up across primary care clinics was attributable to differences in observed patient characteristics, and whether variation was greater than expected by chance after adjustment for patient characteristics (SAS PROC GLIMMIX). In these mixed-effects logistic regression models, we treated center and individual patient characteristics as fixed effects and primary care clinic as a random effect. To facilitate comparisons across demographically diverse populations, all models were adjusted for age, colorectal and breast models were adjusted for race/ethnicity, and colorectal models were also adjusted for sex. Primary care clinic-level variation in the percentage of abnormal screens and percentage with timely initial follow-up is described as the range in the 25th to 75th percentile clinics estimated using a clinic variance component.

\section{RESULTS}

The characteristics of 1,024,566 individuals who underwent screening are reported in Table 2. Breast centers had comparable ages but differed in race/ethnicity. Colorectal centers differed in both age and race/ethnicity. The cervical center showed a smaller proportion screened at ages 60-65 years and included a sizable Hispanic population, as did one of the colorectal centers.

\section{Screening Abnormality Rates}

A range in screening abnormality rates was seen across centers and organ sites (Table 3), with some notable age-related patterns (Appendix Table, available online). For breast cancer screening, abnormality rates were higher for 40-49-year-olds than 50-75-year-olds. Colorectal cancer screening begins at age 50, and abnormality rates were lower for 50-64-year-olds than 65-75-year-olds. For cervical cancer screening, abnormality rates declined with age for all categories of abnormal results, with rates in the younger age group approaching those for breast cancer screening.

\section{Timely Initial Follow-Up of Screening Abnormalities}

The percentage with timely follow-up was highest for breast screening, at $95.6 \%$ (Table 3 ). While abnormality follow-up was lower for both colorectal cancer $(68.1 \%)$ and cervical cancer screening (44.8\%), cervical abnormality follow-up tended to increase with more severe screening abnormalities, with the highest rates for ASC-H/HSIL (52.6 \%).

Time to initial follow-up by center is shown in Figure 1. Time to follow-up was shortest for breast cancer screening and longest for cervical cancer screening. While the breast centers have tightly clustered time-to-follow-up curves, statistically significant variation in time to follow-up was observed across the colorectal centers (log-rank test, $p$ value $<0.001$ ).

\section{Variation in Screening Outcomes Across Primary Care Clinics}

Analyses of clinic-level variation in abnormality rates included 33,236 women from 61 clinics for breast, 27,416 individuals from 37 clinics for colorectal, and 164,772 women from 427 clinics for cervical cancer screening. The variation in abnormality rates across primary care clinics by center is shown in Figure 2a. While median abnormality rates were similar across the breast sites -98.0 IQR (87.5-109.5) for UPenn and 93.7 IQR (81.5-102.9) for Dartmouth-BWH-UPenn had more clinics identified 
Table 2 Characteristics of Screened Populations and Clinics by Cancer Type and PROSPR Center

\begin{tabular}{|c|c|c|c|c|c|c|c|}
\hline \multirow[t]{3}{*}{ Characteristics } & \multicolumn{3}{|l|}{$\begin{array}{l}\text { BREAST } \\
\text { Ages 40-75 }\end{array}$} & \multicolumn{3}{|c|}{$\begin{array}{l}\text { COLORECTAL } \\
\text { Ages 21-65 }\end{array}$} & \multirow{2}{*}{$\begin{array}{l}\text { CERVICAL } \\
\text { Ages 21-65 } \\
\text { New Mexicot }\end{array}$} \\
\hline & \multirow{2}{*}{$\begin{array}{l}\text { UPenn } \\
n=17,479(\%)\end{array}$} & \multirow{2}{*}{$\begin{array}{l}\text { Dartmouth- } \\
\text { BWH } \\
n=; 29,353(\%)\end{array}$} & \multirow{2}{*}{$\begin{array}{l}\text { Vermont } \\
n=50,851(\%)\end{array}$} & \multirow{2}{*}{$\begin{array}{l}\text { Parkland- } \\
\text { UTSW* } \\
n=8553(\%)\end{array}$} & \multirow{2}{*}{$\begin{array}{l}\text { Group } \\
\text { Health } \\
n=18,977(\%)\end{array}$} & \multirow{2}{*}{$\begin{array}{l}\begin{array}{l}\text { Kaiser } \\
\text { Permanente } \dagger\end{array} \\
n=732,023(\%)\end{array}$} & \\
\hline & & & & & & & $n=167,330(\%)$ \\
\hline \multicolumn{8}{|l|}{ Age } \\
\hline $21-29$ & $\mathrm{~N} / \mathrm{A}$ & $\mathrm{N} / \mathrm{A}$ & $\mathrm{N} / \mathrm{A}$ & $\mathrm{N} / \mathrm{A}$ & $\mathrm{N} / \mathrm{A}$ & $\mathrm{N} / \mathrm{A}$ & $44,168(26.4)$ \\
\hline $30-39$ & $\mathrm{~N} / \mathrm{A}$ & $\mathrm{N} / \mathrm{A}$ & $\mathrm{N} / \mathrm{A}$ & $\mathrm{N} / \mathrm{A}$ & $\mathrm{N} / \mathrm{A}$ & $\mathrm{N} / \mathrm{A}$ & $39,912(23.9)$ \\
\hline $40-49$ & $4829(27.6)$ & 8495 (28.9) & $13,159(25.9)$ & $\mathrm{N} / \mathrm{A}$ & $\mathrm{N} / \mathrm{A}$ & $\mathrm{N} / \mathrm{A}$ & $35,656(21.3)$ \\
\hline $50-59$ & $5920(33.9)$ & $10,172(34.7)$ & $18,321(36.0)$ & $5931(69.3)$ & $7876(41.5)$ & $368,378(50.3)$ & $33,685(20.1)$ \\
\hline $60-69$ & $5108(29.2)$ & $8191(28.0)$ & 14,667 (28.8) & $2622(30.7)$ & $8271(43.6)$ & $271,783(37.1)$ & $13,909(8.3)$ \\
\hline $70-75$ & $1622(9.3)$ & $2495(8.5)$ & $4704(9.3)$ & $\mathrm{N} / \mathrm{A}$ & $2830(14.9)$ & $91,862(12.5)$ & N/A \\
\hline \multicolumn{8}{|l|}{ Gender§ } \\
\hline Female & $17,479(100.0)$ & $29,338(100.0)$ & $50,851(100.0)$ & $5647(66.0)$ & $10,160(53.5)$ & $394,130(53.8)$ & $167,330(100.0)$ \\
\hline \multicolumn{8}{|l|}{ Combined Race/ } \\
\hline \multicolumn{8}{|l|}{ Ethnicity\| } \\
\hline White & 9918 (58.6) & $22,473(78.9)$ & $47,666(95.1)$ & $1240(14.5)$ & $14,248(75.1)$ & $383,089(52.3)$ & $70,111(41.9)$ \\
\hline Black & $5523(32.6)$ & $2215(7.8)$ & $151(0.3)$ & $3208(37.5)$ & $701(3.7)$ & $54,724(7.5)$ & 2677 (1.6) \\
\hline Asian/Pacific Islander & $525(3.1)$ & $825(2.9)$ & $416(0.8)$ & $467(5.5)$ & $2029(10.7)$ & $103,686(14.2)$ & $3681(2.2)$ \\
\hline Other & $699(4.1)$ & $76(0.3)$ & $947(1.9)$ & $20(0.2)$ & $562(3.0)$ & $2841(0.4)$ & $20,414(12.2)$ \\
\hline Hispanic & 267 (1.6) & $2900(10.2)$ & $925(1.9)$ & $3589(42.0)$ & $744(3.9)$ & $140,252(19.2)$ & $70,446(42.1)$ \\
\hline Unknown/missing & 547 & 864 & 769 & $29(0.3)$ & $693(3.7)$ & $47,431(6.5)$ & N/A \\
\hline \multicolumn{8}{|l|}{ Insurance Status } \\
\hline Medicaid & $1179(6.8)$ & $1666(5.7)$ & Not available & $648(7.6)$ & $25(0.1)$ & $13,823(1.9)$ & Not available \\
\hline Medicare & $3872(22.2)$ & $6079(20.8)$ & in PROSPR & $801(9.4)$ & $7144(37.6)$ & $231,546(31.6)$ & in PROSPR \\
\hline Commercial/private & $12,302(70.4)$ & $17,315(59.2)$ & Center data & $294(3.4)$ & $11,336(59.7)$ & $486,367(66.4)$ & Center data \\
\hline Other & $115(0.7)$ & $3625(12.4)$ & & $296(3.5)$ & $472(2.5)$ & $238(0.0)$ & \\
\hline Uninsured & $4(0.0)$ & $538(1.8)$ & & $6383(74.6)$ & $0(0.0)$ & $0(0.0)$ & \\
\hline Unknown/missing & 7 & 130 & & $131(1.5)$ & $0(0.0)$ & $49(0.0)$ & \\
\hline Primary care clinics\# & 55 & 37 & & 12 & 28 & 193 & 1021 \\
\hline Primary care providers\# & 567 & 588 & & 208 & 312 & 7277 & 2712 \\
\hline
\end{tabular}

* Only individuals aged 50-64 are included

† Kaiser Permanente includes all primary care providers who performed ambulatory visits from 1/1/2010 through 12/31/2012, rather than providers associated with FIT/FOBT

tOnly individuals aged 60-65 are included in the 60-69 age category

$\$ 38$ individuals at Kaiser Permanente have "unknown" gender, and thus the total for Gender in the KP column does not equal the KP total

IIFor cervical, estimates are calculated from percentages based on the US Census for females aged 21-65 years in calendar year 2012

IFor individuals with multiple insurance types, the following hierarchy was used: 1) Medicaid, 2) Medicare, 3) Commercial/private, 4) Other, 5)

Uninsured (includes uninsured and those enrolled in a medical assistance charity program for the uninsured), 6) Unknown/missing

\#Number of non-missing clinics and providers. For cervical cancer, clinics and providers performing tests/procedures are reported rather than primary care clinics and providers

as outliers on both the upper and lower ends of the spectrum. Clinic-level abnormality rates differed somewhat among colorectal sites (medians of $32.1 \mathrm{IQR}(27.6-46.4)$ for Parkland-UTSW and 49.6 IQR (37.5-56.3) for GH). The cervical center had more outliers than did breast or colorectal centers. Clinic-level variation in the percentage of abnormal screening exams receiving timely follow-up was least pronounced for breast cancer screening, intermediate for colorectal cancer screening, and most pronounced for cervical cancer screening (Fig. 2b).

Analyses of primary care clinic-level variation in the percentage with timely initial follow-up included 3144 women from 51 clinics for breast, 966 individuals from 29 clinics for colorectal, and 6697 women from 207 clinics for cervical cancer screening. There was significant variation across primary care clinics for both the percentage with an abnormal screen (25th to 75th percentile for breast, 8.5 to $10.3 \%$; for colorectal, 3.0 to $4.8 \%$; for cervical, 6.3 to $9.9 \%$ ) and the percentage having timely follow-up (25th to 75 th percentile for breast, 90.2 to $95.8 \%$; for colorectal, 43.4 to $52 \%$; for cervical, 29.6 to $61.4 \%$ ).

\section{DISCUSSION}

Our study characterizes the variation observed across health care settings and patient populations in screening abnormalities for breast, colorectal, and cervical cancer screening and their timely follow-up - an important process measure for effective cancer screening. ${ }^{14}$ Timely follow-up was most frequently achieved for breast cancer screening, where the most severe abnormalities (BI-RADS 4 or 5) had 88.9 to $100 \%$ follow-up at 3 months. In contrast, for colorectal cancer screening-where only two-thirds of individuals with FOBT/FIT + received follow-up within 90 days - there is ample opportunity for improvement. Likewise, among women with cervical screening abnormalities for whom receipt of guideline-concordant colposcopy within 3 months was expected, only 42.9 to $52.6 \%$ received such care.

Screening abnormality rates observed across PROSPR are consistent with prior studies, ${ }^{12,15-22}$ yet the cross-organ perspective highlights the higher abnormality rate for breast cancer screening ( $10.5 \%$ of individuals screened) relative to colorectal cancer screening using FOBT/FIT (4.7 \% of individuals screened) or cervical cancer screening $(7.9 \%$ of 


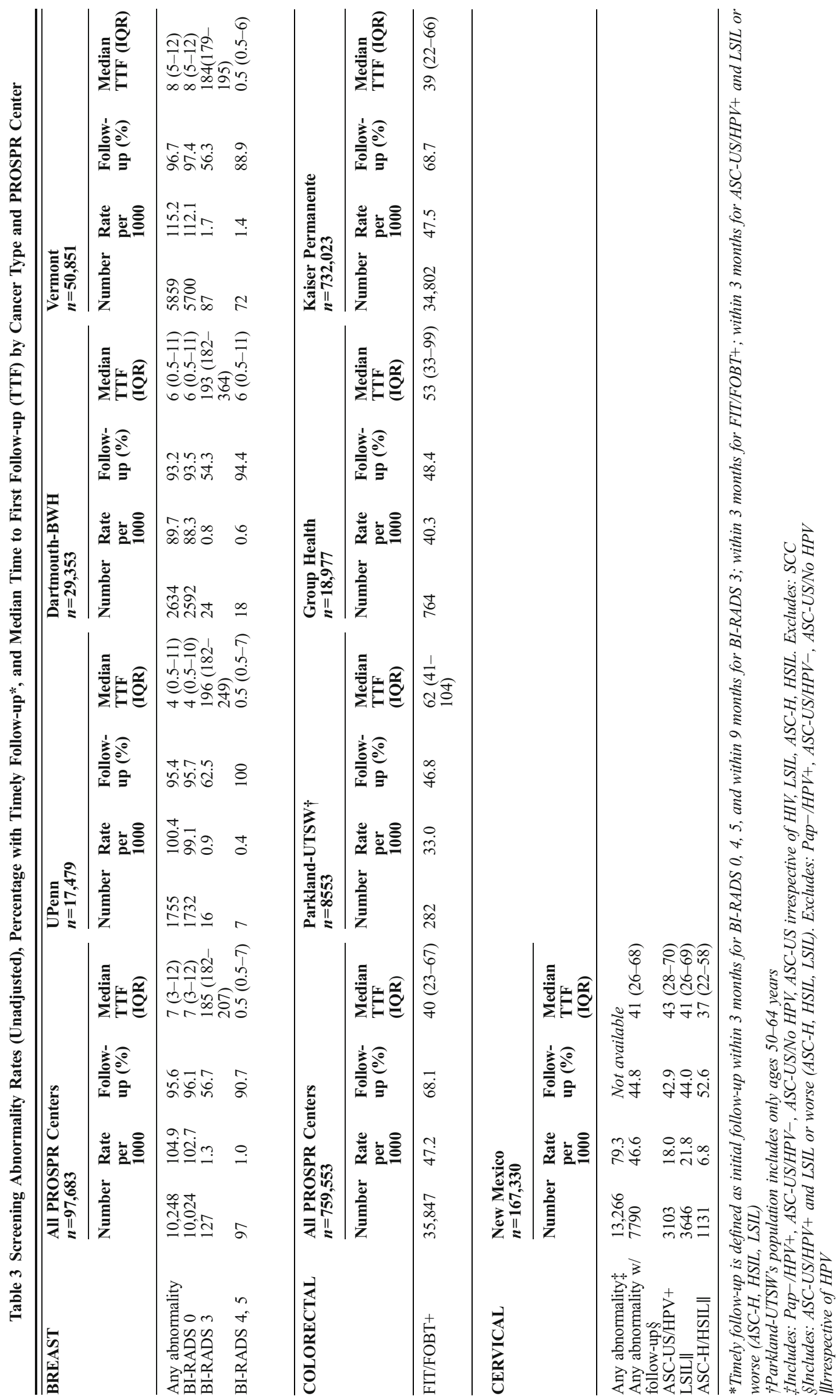




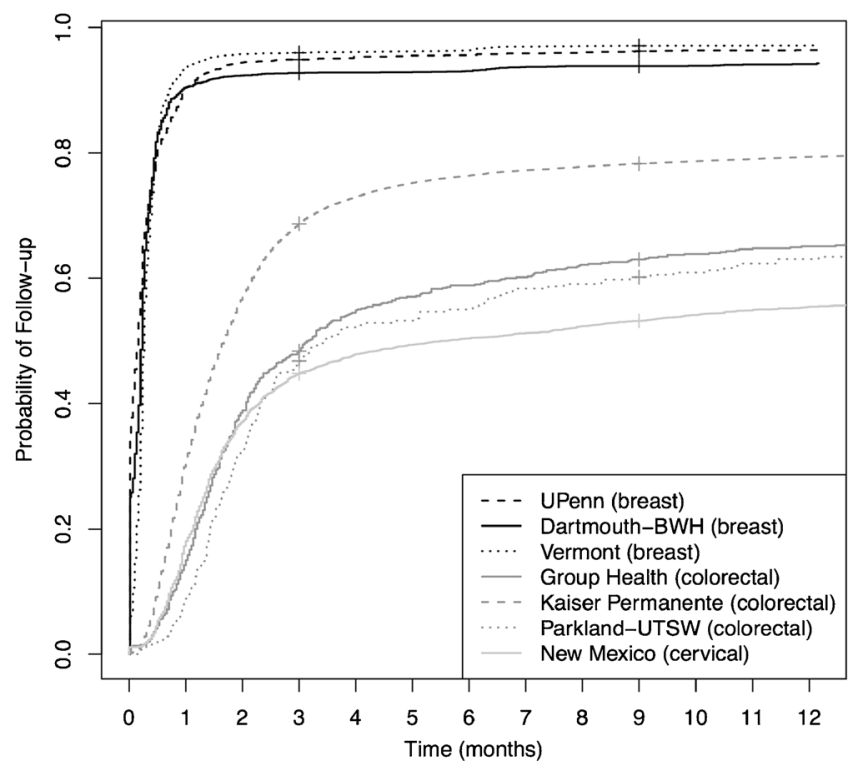

Figure 1 Kaplan-Meier curves for time to first follow-up by organ and PROSPR Center. Ticks $(+)$ are shown at 3 and 9 months.

individuals screened). Differences in rates of colorectal cancer screening abnormalities may reflect differences in the proportion of individuals undergoing first-time versus repeat FOBT/ FIT screening. Since colorectal adenomas are detected more frequently in men and older patients, Parkland-UTSW's younger population with a higher proportion of women would be expected to have a lower abnormal test rate than GH and KP.

Other factors may contribute to variation in the observed organ-specific abnormality rates, including the mix of breast imaging modalities with different operating characteristics, the sensitivity of different types of fecal occult blood tests, and the subjectivity of radiologist and cervical cytology interpretations, as well as differences in laboratory processing procedures. While UPenn was an early adopter of digital breast tomosynthesis, their abnormality rate was intermediate, between the rates observed for Dartmouth-BWH and VT. The health systems also differed in the types of fecal tests (FOBT and FIT) and processors used during the years studied. ${ }^{7}$ The lower abnormality rate found for GH in comparison to KP could reflect the use of the FOBT (used at some GH clinics through 2011), which is more specific and less sensitive than FIT (used at KP). ${ }^{23}$ For cervical cancer screening, the higher variation in abnormality rates may be attributable to the diverse populations served at specific clinics and to the fact that we did not restrict analysis to primary care clinics or providers.

From a primary care population health management perspective, variation in timely screening abnormality follow-up is concerning, and may be influenced by patient and system characteristics as well as differences in the underlying complexity of coordinating the next steps in clinical care. ${ }^{24}$ Inherent differences in the severity of abnormalities detected and the type of follow-up required may also affect timely followup. For example, the lower follow-up rates for colorectal cancer screening abnormalities may be due to the perceived
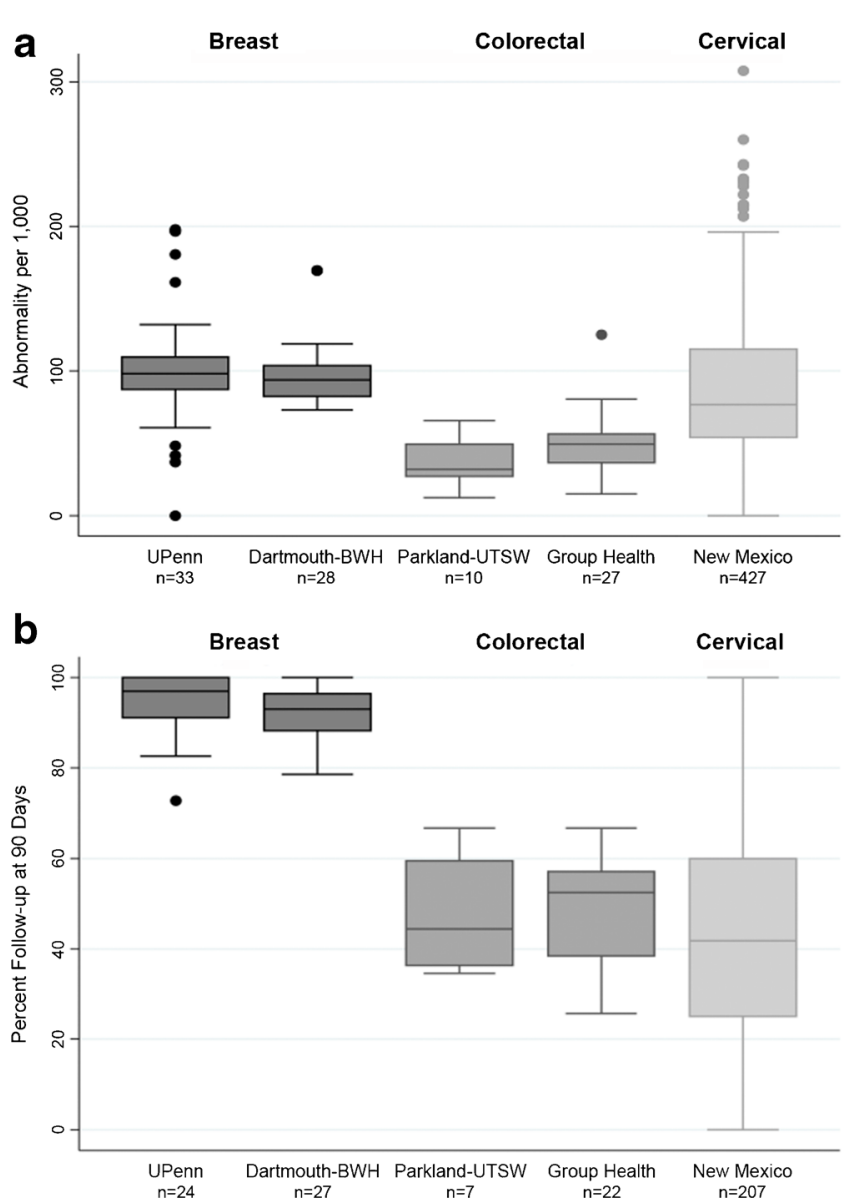

Figure 2 Box plots of clinic-level screening outcomes by organ and PROSPR Center, with number of clinics included shown for each center. (a) Screening abnormality rates. Limited to clinics with 25 or more screening exams. Cervical includes: Pap-/HPV+, ASC-US/ HPV-, ASC-US/No HPV, ASC-US/HPV+, LSIL or worse (any HPV result). (b) Percentage with timely follow-up*. Limited to clinics with 11 or more abnormal screening exams. Cervical includes: ASC-US/ HPV+ and LSIL or worse (any HPV result). *Timely follow-up is defined as initial follow-up within 3 months for BI-RADS 0,4 , and 5 , and within 9months for BI-RADS 3; within 3 months for FIT/ FOBT+; and within 3 months for ASC-US/HPV+ and LSIL or worse (ASC-H, HSIL, LSIL).

inconvenience and invasive nature of the procedures involved. Patients undergoing colonoscopy typically need to take time off work, which may pose a barrier especially for lowerincome individuals. Preparation for colonoscopy also includes dietary restriction, bowel preparation, and sedation in an inpatient or same-day surgical environment. Furthermore, patients with a positive FOBT/FIT must transition from the primary care setting to gastrointestinal (GI) specialty care. This requires an interface - $\mathrm{a}$ transfer of role/responsibility between primary care and specialist. ${ }^{25}$ Similar challenges are encountered for follow-up of cervical screening abnormalities, as specialist referral for diagnostic colposcopy is often required. In contrast, the initial follow-up for breast cancer screening abnormalities often entails additional imaging, which may be completed in the same setting and often on the same day.

The nature of the populations served may also affect the timeliness of follow-up care. ${ }^{26-30}$ At Parkland-UTSW, a 
safety-net setting, GI clinics must handle all symptomatic uninsured patients who walk in through the emergency department, which may overwhelm the capacity of GI clinics to perform timely colonoscopy follow-up.

Health care organizations' capacity, integration of services, and outreach may also influence the timeliness of follow-up. During the time interval studied, the colorectal center with the most favorable follow-up (KP) began implementing benchmarks and pathways designed to promote colonoscopy follow-up. These include a goal of 14 days for follow-up of a positive FIT with dedicated staff to directly schedule diagnostic colonoscopies by phone, email, and/or standard mail (KPsouthern region), and a goal of $80 \%$ diagnostic colonoscopy performance within 1 month of a positive FIT (KP-northern region). ${ }^{31}$ The provision of monthly reports on time to followup for GI physicians and clinical leaders at KP likely also contributes to higher follow-up rates.

Federal policies and regulations that encourage timely cancer screening may affect screening follow-up well beyond individual patient and health system factors. Such policies exist for breast and cervical cancer screening, but are nonexistent for colorectal cancer screening, for which we found the greatest variation in timely follow-up. Since its reauthorization in 1999, the national Mammography Quality Standards Act (MQSA; 42 U.S.C. 263b), which regulates breast cancer screening quality and the use of the Breast Imaging-Reporting and Data System (BI-RADS), ${ }^{32}$ has promoted timely followup of screening abnormalities. Importantly, the MQSA requires that health care facilities provide patients with written results of their mammograms in language that is easy to understand, and also requires reporting of breast cancer screening results to primary care providers. These regulations may partly explain the smaller variation in follow-up rates across centers and clinics for breast cancer screening compared to colorectal and cervical cancer screening.

Prior to enactment of the MQSA, the Breast and Cervical Cancer Mortality Prevention Act of 1990 (Public Law 101354) established the Centers for Disease Control and Prevention's National Breast and Cervical Cancer Early Detection Program, which provides screening and diagnostic follow-up to low-income and under- or uninsured women, set targets for timely follow-up of screening abnormalities ( $80 \%$ complete diagnostic evaluation within 90days), and ties funding to meeting those targets. ${ }^{33}$ The act also funded public education designed to increase public awareness of the benefits of early detection, which may contribute to timely follow-up by patients with screening abnormalities.

Although colorectal cancer screening is not regulated by federal policies, it is encouraged through national clinical guidelines and the National Committee for Quality Assurance's Healthcare Effectiveness Data and Information Set (HEDIS) for health plan quality reporting. ${ }^{34}$ However, HEDIS cancer screening-related quality measures address the provision of screening services rather than timely follow-up of abnormalities.
While the diversity of settings represented within PROSPR provides rich opportunities for comparative studies, some limitations warrant mention. First, cross-organ comparisons may be hampered by the different care settings represented across organ sites, with some settings affected to a greater degree by potential out-of-system care. ${ }^{35}$ Second, the data available through each center differ in source and quality. The two statewide registries provide strength in their inclusion of more heterogeneous populations than may be seen among the insured who are cared for within integrated health systems or primary care networks, but have more limited access to detailed clinical information. However, even when electronic health record data are available, challenges remain in creating comparable data across centers given the differences among systems and the lack of structured data for covariates of interest in studying screening processes of care (e.g., risk factors) ${ }^{36,37}$ Finally, PROSPR's common data elements capture care that was delivered but do not include information on scheduled care.

Our study underscores the potential for using PROSPR data to gain insight into factors affecting timely follow-up of screening abnormalities and for understanding whether timeliness of follow-up results in different clinical outcomes. Gaining insight into best screening processes of care within PROSPR will require multi-level investigation of patient, provider, clinic, and health system factors, and entails assembling health system information not readily available through electronic health records alone. ${ }^{38,39}$ Clinical care pathways regarding communication of test results is one such area where the consortium is actively working to document current practices. These data will enable evaluation of the role of communication at care interfaces in the timely follow-up of screening abnormalities. This should advance systems approaches to improving the quality of cancer care, which are already gaining traction. ${ }^{40}$ They also allow investigation into whether differences in time to follow-up result in meaningful differences in disease outcomes; such data can inform evidencebased metrics for follow-up guidelines. Other features of the health system and the legal and regulatory environments in which care is provided must also be considered, including the extent to which clinics participating in PROSPR have received National Council on Quality Assurance recognition as a patient-centered medical home. ${ }^{41}$ Through focused comparative effectiveness studies, the variation documented in rates of screening abnormalities and follow-up within PROSPR may help guide the development and implementation of health care delivery interventions and policies with the potential to improve population health.

Acknowledgments: The authors thank the participating PROSPR Research Centers for the data they have provided for this study. A list of the PROSPR investigators and contributing research staff are provided at: http://healthcaredelivery.cancer.gov/prospr/.

Corresponding Author: Anna N. A. Tosteson, ScD; Geisel School of Medicine at Dartmouth, Lebanon, NH, USA (e-mail: Anna.Tosteson@Dartmouth.edu). 


\section{Compliance with Ethical Standards:}

Conflict of Interest: The authors declare no conflicts of interest.

Funders: This work was supported by the National Cancer Institute (NCI)-funded Population-based Research Optimizing Screening through Personalized Regimens (PROSPR) consortium (grant numbers U01CA163304 to M.T., W.B.; U54CA163303 to D.L.W., B.S.; U54CA163307 to A.N.A.T., T.O., J.H.; U54CA163313 to K.A., M.S.; U54CA163308 to C.S.S., E.H.; U54CA163308-04S1 to C.S.S., J.A.T.; U54CA163261 to C.R.; U54CA163261-04S1 to J.C., A.K.; U54CA163262 to A.G.Z., D.C., C.D., T.L.; U54CA163262-04S1 to D.C., M.S.; and U54CA164336 to C.W.). The content of this manuscript is solely the responsibility of the authors and does not necessarily represent the official views of the Department of Veterans Affairs or the United States government.

\section{REFERENCES}

1. Koh HK, Sebelius KG. Promoting prevention through the affordable care Act. N Engl J Med. 2010;363(14):1296-9.

2. U.S. Preventive Services Task Force. Screening for breast cancer: U.S. Preventive services task force recommendation statement. Ann Intern Med. 2009;151(10):716-26. W-236.

3. Moyer VA, Force USPST. Screening for cervical cancer: US preventive services task force recommendation statement. Ann Intern Med. 2012;156(12):880-U891.

4. U.S. Preventive Services Task Force. Screening for colorectal cancer: U.S. Preventive services task force recommendation statement. Ann Intern Med. 2008; 149(9):627-37.

5. Beaber EF, Kim JJ, Schapira MM, et al. Unifying screening processes within the PROSPR consortium: a conceptual model for breast, cervical, and colorectal cancer screening. J Natl Cancer Inst. 2015;107(6):djv120.

6. Onega T, Beaber EF, Sprague BL, et al. Breast cancer screening in an era of personalized regimens: a conceptual model and National Cancer Institute initiative for risk-based and preference-based approaches at a population level. Cancer. 2014;120(19):2955-64.

7. Tiro JA, Kamineni A, Levin TR, et al. The colorectal cancer screening process in community settings: a conceptual model for the populationbased research optimizing screening through personalized regimens consortium. Cancer Epidemiol Biomarkers Prev. 2014;23(7):1147-58.

8. Zapka JG, Taplin SH, Solberg LI, Manos MM. A framework for improving the quality of cancer care: the case of breast and cervical cancer screening. Cancer Epidemiol Biomarkers Prev. 2003;12(1):4-13.

9. Rittenhouse DR, Shortell SM, Fisher ES. Primary care and accountable care-two essential elements of delivery-system reform. N Engl J Med. 2009;361(24):2301-3.

10. O'Malley AS, Tynan A, Cohen GR, Kemper N, Davis MM. Coordination of care by primary care practices: strategies, lessons and implications. Res Brief. 2009; 12:1-16.

11. Shih A, Davis K, Schoenbaum S, et al. Organizing the U.S. health care delivery system for high performance.: Commonwealth Fund. 2008.

12. Battaglia TA, Santana MC, Bak S, et al. Predictors of timely follow-up after abnormal cancer screening among women seeking care at urban community health centers. Cancer. 2010;116(4):913-21.

13. Yabroff KR, Freedman A, Brown ML, et al. Trends in abnormal cancer screening results in the United States of America. J Med Screen. 2007; 14(2):67-72.

14. Taplin SH, Yabroff KR, Zapka J. A multilevel research perspective on cancer care delivery: the example of follow-up to an abnormal mammogram. Cancer Epidemiol Biomarkers Prev. 2012;21(10):1709-15.

15. Benard VB, Howe W, Royalty $\mathbf{J}$, et al. Timeliness of cervical cancer diagnosis and initiation of treatment in the national breast and cervical cancer early detection program. J Womens Health (Larchmt). 2012;21(7):776-82.

16. Chang SW, Kerlikowske K, NapolesSpringer A, et al. Racial differences in timeliness of follow-up after abnormal screening mammography. Cancer. 1996;78(7):1395-402.

17. Eheman CR, Benard VB, Blackman D, et al. Breast cancer screening among low-income or uninsured women: results from the national breast and cervical cancer early detection program, July 1995 to march 2002 (united states). Cancer Causes Control. 2006;17(1):29-38.

18. Elit L, Krzyzanowska M, Saskin R, et al. Sociodemographic factors associated with cervical cancer screening and follow-up of abnormal results. Can Fam Physician. 2012;58(1):E22-31.
19. Etzioni DA, Yano EM, Rubenstein LV, et al. Measuring the quality of colorectal cancer screening: the importance of follow-up. Dis Colon Rectum. 2006;49(7):1002-10.

20. Major D, Bryant H, Delaney M, et al. Colorectal cancer screening in Canada: results from the first round of screening for five provincial programs. Curr Oncol. 2013;20(5):252-7.

21. Perez-Stable EJ, Afable-Munsuz A, Kaplan CP, et al. Factors influencing time to diagnosis after abnormal mammography in diverse women. J Womens Health (Larchmt). 2013;22(2): 159-66.

22. Rosenberg RD, Haneuse SJ, Geller BM, et al. Timeliness of follow-up after abnormal screening mammogram: variability of facilities. Radiology. 2011;261(2):404-13.

23. Wong CK, Fedorak RN, Prosser CI, et al. The sensitivity and specificity of guaiac and immunochemical fecal occult blood tests for the detection of advanced colonic adenomas and cancer. Int $\mathrm{J}$ Colorectal Dis. 2012;27(12): 1657-64.

24. Richards MA, Westcombe AM, Love SB, Littlejohns P, Ramirez AJ. Influence of delay on survival in patients with breast cancer: a systematic review. Lancet. 1999;353(9159):1119-26.

25. Beaber EF, Kim JJ, Scapira MM, et al. Unifying screening processes within the PROSPR consortium: A conceptual model for breast, cervical, and colorectal cancer screening. Under Rev. 2015.

26. Eggleston KS, Coker AL, Das IP, Cordray ST, Luchok KJ. Understanding barriers for adherence to follow-up care for abnormal pap tests. J Womens Health (Larchmt). 2007;16(3):311-30.

27. Elmore JG, Nakano CY, Linden HM, et al. Racial inequities in the timing of breast cancer detection, diagnosis, and initiation of treatment. Med Care. 2005;43(2):141-8.

28. Goldman LE, Walker R, Hubbard $\mathbf{R}$, Kerlikowske $\mathbf{K}$, Breast CS. Timeliness of abnormal screening and diagnostic mammography follow-up at facilities serving vulnerable women. Med Care. 2013;51(4):307-14.

29. Karliner LS, Ma L, Hofmann M, Kerlikowske K. Language barriers, location of care, and delays in follow-up of abnormal mammograms. Med Care. 2012;50(2):171-8.

30. Turner B, Myers RE, Hyslop T, et al. Physician and patient factors associated with ordering a colon evaluation after a positive fecal occult blood test. J Gen Intern Med. 2003;18(5):357-63.

31. Chubak J, Garcia MP, Burnett-Hartman A, et al. Time to colonoscopy after positive fecal blood test in four U.S. health care systems. Under Rev. 2015.

32. D'Orsi CJSE, Mendelson EB, Morris EA, et al. ACR BI-RADS $®$ atlas, breast imaging reporting and data system. Reston: American College of Radiology; 2013.

33. Saslow D, Solomon D, Lawson HW, et al. American cancer society, American society for colposcopy and cervical pathology, and american society for clinical pathology screening guidelines for the prevention and early detection of cervical cancer. CA Cancer $\mathrm{J}$ Clin. 2012;62(3): 147-72.

34. National Committee for Quality Assurance (NCQA). HEDIS and Performance Measurement. 2015; http://www.ncqa.org/HEDISQualityMeasurement.aspx, 2015. Accessed May 1, 2015.

35. McDonald KM, Schultz E, Chapman T, et al. Prospects for Care Coordination Measurement Using Electronic Data Sources (Prepared by Stanford University under subcontract to Battelle on Contract No. 290-040020-AHRQ SQI-II.) AHRQ Publication No. 12-0014-EF. Rockville, MD: Agency for Healthcare Quality and Research. 2012.

36. Chan KS, Fowles JB, Weiner JP. Review: electronic health records and the reliability and validity of quality measures: a review of the literature. Med Care Res Rev. 2010;67(5):503-27.

37. Roth CP, Lim YW, Pevnick JM, Asch SM, McGlynn EA. The challenge of measuring quality of care from the electronic health record. Am J Med Qual. 2009;24(5):385-94.

38. Cleary PD, Gross CP, Zaslavsky AM, Taplin SH. Multilevel interventions: study design and analysis issues. J Natl Cancer Inst Monogr. 2012;2012(44):49-55.

39. Zapka JM, Edwards HM, Chollette V, Taplin SH. Follow-up to abnormal cancer screening tests: considering the multilevel context of care. Cancer Epidemiol Biomark Prev. 2014;23(10): 1965-73.

40. Yabroff KR, Zapka J, Klabunde CN, et al. Systems strategies to support cancer screening in US primary care practice. Cancer Epidemiol Biomark Prev. 2011;20(12):2471-9.

41. Sarfaty M, Wender R, Smith R. Promoting cancer screening within the patient centered medical home. CA Cancer J Clin. 2011;61(6):397408. 